\title{
A importância da conciliação medicamentosa em hospitais brasileiros
}

\author{
The importance of medication reconciliation in brazilian hospitals \\ La importancia de la conciliación de medicamentos en los hospitales brasileños
}

Weilla Patricia Cordeiro Silva

ORCID: https://orcid.org/0000-0003-3544-7577

Universidade Federal do Pará, Brasil

E-mail: weillacoordeiro1@gmail.com

Aline Farias Ribeiro

ORCID: https://orcid.org/0000-0002-9240-0532

Universidade Federal do Pará, brasil

E-mail: afariasribeiro@hotmail.com

José Eduardo Gomes Arruda

ORCID: https://orcid.org/0000-0002-8331-5563

Universidade Federal do Pará, Brasil

E-mail: josearruda@ufpa.br

\begin{abstract}
Resumo
A conciliação de medicamentos relaciona-se diretamente com a segurança das pessoas nos diferentes níveis de assistência à saúde em que os medicamentos são utilizados como principais tecnologias para a cura, diagnóstico e o controle de doenças. Foi realizada uma revisão integrativa da literatura com o objetivo de conhecer acerca da importância da conciliação medicamentosa para garantir a segurança do paciente hospitalizado. O levantamento bibliográfico abrangeu estudos somente em português, publicados entre 2016 a 2021, em periódicos científicos e que estivessem adequados ao tema proposto para o trabalho, a pesquisa foi realizada no mês de novembro de 2021 nas bases de dados PubMed, SCIELO, portal CAPES, LILACS, Google acadêmico e BVS, utilizando os seguintes descritores: "conciliação medicamentosa", "hospital", "Brasil" e "segurança do paciente". Sendo identificados, após análise, 13 artigos que compuseram a amostra do estudo. Os resultados apontam que na maior parte dos artigos analisados, a conciliação medicamentosa em pacientes hospitalizados, apresenta-se como fundamental para a segurança do mesmo. Em todos conseguimos perceber a importância de se monitorar as prescrições e trabalhar em conjunto com a equipe multiprofissional. Portanto, foi de extrema relevância a realização desta revisão integrativa para a comprovação que o cuidado farmacêutico é uma ferramenta positiva para a saúde da população. Contudo, observou-se carência em estudos sobre a conciliação medicamentosa em hospitais brasileiros.
\end{abstract}

Palavras-chave: Reconciliação de medicamentos; Serviço de farmácia hospitalar; Segurança do paciente.

\begin{abstract}
The reconciliation of medications is directly related to the safety of people at different levels of health care in which medications are used as the main technologies for the cure, diagnosis and control of diseases. An integrative literature review was carried out to learn about the importance of medication reconciliation to ensure the safety of hospitalized patients. The bibliographic survey covered studies only in Portuguese, published between 2016 and 2021, in scientific journals and that were adequate to the theme proposed for the work, the research was carried out in November 2021 in the databases PubMed, SCIELO, CAPES portal, LILACS, Google academic and VHL, using the following descriptors: "drug reconciliation", "hospital", "Brazil" and "patient safety". After analysis, 13 articles that comprised the study sample were identified. The results show that in most of the articles analyzed, medication reconciliation in hospitalized patients is fundamental for their safety. In all of them, we were able to see the importance of monitoring prescriptions and working together with the multidisciplinary team. Therefore, it was extremely important to carry out this integrative review to prove that pharmaceutical care is a positive tool for the health of the population. However, there was a lack of studies on medication reconciliation in Brazilian hospitals.
\end{abstract}

Keywords: Medication reconciliation; Pharmacy service hospital; Patient safety.

\section{Resumen}

La conciliación de medicamentos está directamente relacionada con la seguridad de las personas en los diferentes niveles de atención de la salud en los que los medicamentos se utilizan como principales tecnologías para la cura, el diagnóstico y el control de enfermedades. Se realizó una revisión integradora de la literatura con el fin de conocer la importancia de la conciliación de la medicación para garantizar la seguridad de los pacientes hospitalizados. El relevamiento bibliográfico abarcó estudios solo en portugués, publicados entre 2016 a 2021, en revistas científicas y que fueron adecuados a la temática propuesta para el trabajo, la investigación se realizó en noviembre de 2021 en las 
bases de datos PubMed, SCIELO, portal CAPES, LILACS, Google académico y BVS, utilizando los siguientes descriptores: "conciliación de medicamentos", "hospital", "Brasil" y "seguridad del paciente". Tras el análisis, se identificaron 13 artículos que componían la muestra de estudio. Los resultados muestran que, en la mayoría de los artículos analizados, la conciliación de la medicación en los pacientes hospitalizados es fundamental para su seguridad. En todos ellos pudimos ver la importancia del seguimiento de las prescripciones y el trabajo conjunto con el equipo multidisciplinar. Por ello, fue de suma importancia realizar esta revisión integradora para demostrar que la atención farmacéutica es una herramienta positiva para la salud de la población. Sin embargo, faltaron estudios sobre conciliación de medicamentos en los hospitales brasileños.

Palabras clave: Conciliación de medicamentos; Servicio de farmacia en hospital; Seguridad del paciente.

\section{Introdução}

Os estudos mostram que aproximadamente $60 \%$ dos erros de medicação ocorrem durante a transição de nível de assistência à saúde, mais especificamente em prescrições médicas feitas na internação ou na alta hospitalar. Estima-se que $27 \%$ dos erros das prescrições hospitalares ocorrem devido à falta de informações no momento da admissão. De acordo com o relatório do instituto de medicina preventiva de erros de medicação, em média, o paciente hospitalizado está propício a no mínimo um erro de medicação por dia (Silva et al. 2021). A conciliação de medicamentos (CM) é um serviço realizado pela equipe multidisciplinar, em que há a comparação dos medicamentos utilizados previamente e dos medicamentos prescritos. Possui elevada importância clínica para os pacientes, uma vez que aumenta a segurança por promover redução considerável de potenciais erros de medicação e diminuir as divergências encontradas entre os medicamentos já utilizados e as prescrições hospitalares realizadas no ato da internação (Fernandes et al. 2021).

Os erros de medicação são um dos principais fatores de morbidade nos pacientes acolhidos nos hospitais, podendo ser cometido por todos os membros da equipe e em qualquer etapa do processo incluindo a admissão do paciente no hospital, momento crucial para coleta de informações sobre terapia pregressa e hábitos de vida. Essa falha pode resultar em inadequação da terapia medicamentosa, consequentemente a falha ou comprometimento terapêutico durante o período de internação. Sendo a conciliação medicamentosa um meio indispensável para prevenção desses erros (Magalhães, 2017; Santos et al, 2019).

A CM é entendida como uma ferramenta estratégica e prática no qual se obtém uma lista de medicamentos completa de uso do paciente (nome, a dose, a frequência de uso, via de administração, dados sobre anamnese, entre outras) onde pode ser adotado, a partir desta, ajustes da terapia no período de transição do ambiente domiciliar para o hospitalar, ou até mesmo, em transferências hospitalares internas, com o objetivo de minimizar discrepâncias da prescrição como omissões, duplicações, interações medicamentosas e erros de dosagem (Souza et al., 2018). Diversas organizações internacionais, incluindo a Organização Mundial de Saúde (OMS), incentivam a realização da CM como uma forma de reduzir e prevenir os eventos adversos a medicamentos uma vez que pacientes com discrepâncias registradas, podem apresentar maior ocorrência de eventos adversos (Silva et al. 2021).

O processo de implantação dessa prática tem sido um grande desafio para os hospitais, pois requer uma interação estreita entre as equipes que fazem parte do processo, pois a farmacoterapia tem um caráter multiprofissional. Estudos têm demonstrado que o processo de CM contribuí significativamente na prevenção de feitos adversos a medicamentos e na diminuição das discrepâncias nas prescrições, colaborando para a segurança do paciente (Graça et al., 2018). A CM visa garantir que todas as vezes que o paciente seja deslocado de um setor de cuidado a saúde para outro, as informações acerca dos medicamentos utilizados, sejam repassadas ao mesmo tempo e de forma acurada e completa. No cenário hospitalar, a conciliação pode se configurar como um processo multidisciplinar a ser realizado por farmacêuticos, médicos e/ou enfermeiros no momento da admissão, da transferência entre duas unidades clínicas e da alta (Silvestre \& Júnior, 2018; Tavares, 2019).

Buscando uma melhor segurança no tratamento durante o período de internação, o acompanhamento de uma equipe multiprofissional é indispensável, no qual o farmacêutico tem um importante papel. Este está envolvido diretamente no 
rastreamento e monitoramento do tratamento medicamentoso, com intuito de contribuir na resolução de problemas que interferem na clínica do paciente. Por isso, a prática da CM é uma ferramenta chave para prevenção de eventos adversos e melhora no prognóstico clínico (Fernandes et al. 2021).

Observa-se que existe a necessidade de ampliação do conhecimento sobre a CM, devido haver lacunas existentes na literatura no que tange a mesma. Apesar de esta prática ser considerada prioritária para a segurança dos pacientes, a implantação da conciliação de forma efetiva e sustentada é uma meta a ser alcançada no cenário brasileiro. (Silvestre \& Júnior, 2018). Dessa forma, torna-se de grande importância a presença de um farmacêutico no rastreio de problemas relacionados a medicamentos junto a equipe multiprofissional, melhorando a qualidade de vida dos pacientes, otimizando a terapia e diminuindo os custos hospitalares.

O farmacêutico é um profissional que esta envolvido na segurança do paciente, a qual consiste na redução do risco de danos desnecessários associados à assistência em saúde até um mínimo aceitável. No Brasil, mesmo após a Portaria nº 529 de $1^{\circ}$ de abril de 2013, observa-se a escassez de publicações sobre erros de medicamentos e segurança do paciente em âmbito nacional (Tavares, 2019; Batista et al. 2021). Por esta razão, implantar um protocolo de conciliação medicamentosa, visando à segurança dos pacientes hospitalizados, é necessário. Logo, esta revisão integrativa objetiva ampliar os conhecimentos acerca da importância da CM na segurança do paciente que se encontra em um hospital.

\section{Metodologia}

O presente estudo consiste em uma revisão integrativa de literatura, a qual permite identificar lacunas que precisam ser preenchidas com a realização de novos estudos. Esta revisão discorre sobre a CM para a promoção da segurança de paciente hospitalizado.

Para realizar essa revisão integrativa, considerou-se o estudo de Batista et al. (2021), sendo percorridas as seguintes etapas: definição do tema, elaboração da pergunta norteadora, estabelecimento de critérios de busca ou amostragem na literatura, definição das informações extraídas dos artigos (coleta de dados), análise crítica dos artigos incluídos, discussão e apresentação dos resultados.

Para nortear a revisão integrativa, elaborou-se a seguinte questão: Qual a importância da conciliação medicamentosa para a segurança dos pacientes hospitalizados? A pesquisa foi realizada no mês de novembro de 2021 nas bases de dados descritas abaixo, utilizando os Descritores de Ciências da Saúde (DeCS). Os descritores selecionados foram "conciliação medicamentosa", "hospital", "Brasil” e "segurança do paciente". Algumas vezes foi utilizado o operador booleano "AND”.

As pesquisas foram realizadas utilizando as bases de dados: PubMed, Scientific Eletronic Library (Scielo), LILACS, Google Acadêmico, Portal de Periódicos da CAPES e Biblioteca Virtual em Saúde (BVS). Utilizou-se como critérios de inclusão artigos científicos com resumo e texto completos disponíveis eletronicamente, escritos em português, publicados de 2016 a 2021 , em periódicos científicos e que estivessem adequados ao tema proposto para o trabalho.

Foram excluídos do estudo, artigos que não abordaram, em conjunto, o tema: conciliação medicamentosa realizada em hospitais brasileiros, artigos que abordaram esses aspectos, porém, em outras locais, que não o hospitalar; artigos duplicados nas bases de dados; artigos que não estavam disponíveis na íntegra; que não estavam em português; monografias; teses; dissertações e outros, além de artigos com acesso restrito a pagamento.

O processo de seleção de trabalhos para o presente artigo foi realizado por meio de revisão por pares, de forma independente. As divergências foram discutidas entre os dois autores até se obter um consenso dos artigos que, de fato, seriam selecionados e analisados. 
Dos trabalhos incluídos nesta revisão integrativa, foram extraídas as seguintes informações: autoria, título, local de busca, tipo de pesquisa, descritores, ano de publicação e conclusões/recomendações.

\section{Resultados e Discussão}

Na busca foram encontrados 262 trabalhos (5 na BVS, 3 no portal CAPES, nenhum na SCIELO, 4 na LILACS, 2 na PUBMED e 248 no Google acadêmico). Após as diferentes etapas, foram selecionados para esta revisão 13 artigos científicos, conforme fluxograma apresentado na Figura 1. Tendo em vista os trabalhos selecionados, o quadro 1 sumariza os dados relativos aos artigos incluídos nesta revisão.

Figura 1: Fluxograma da busca de artigos nas bases de dados.

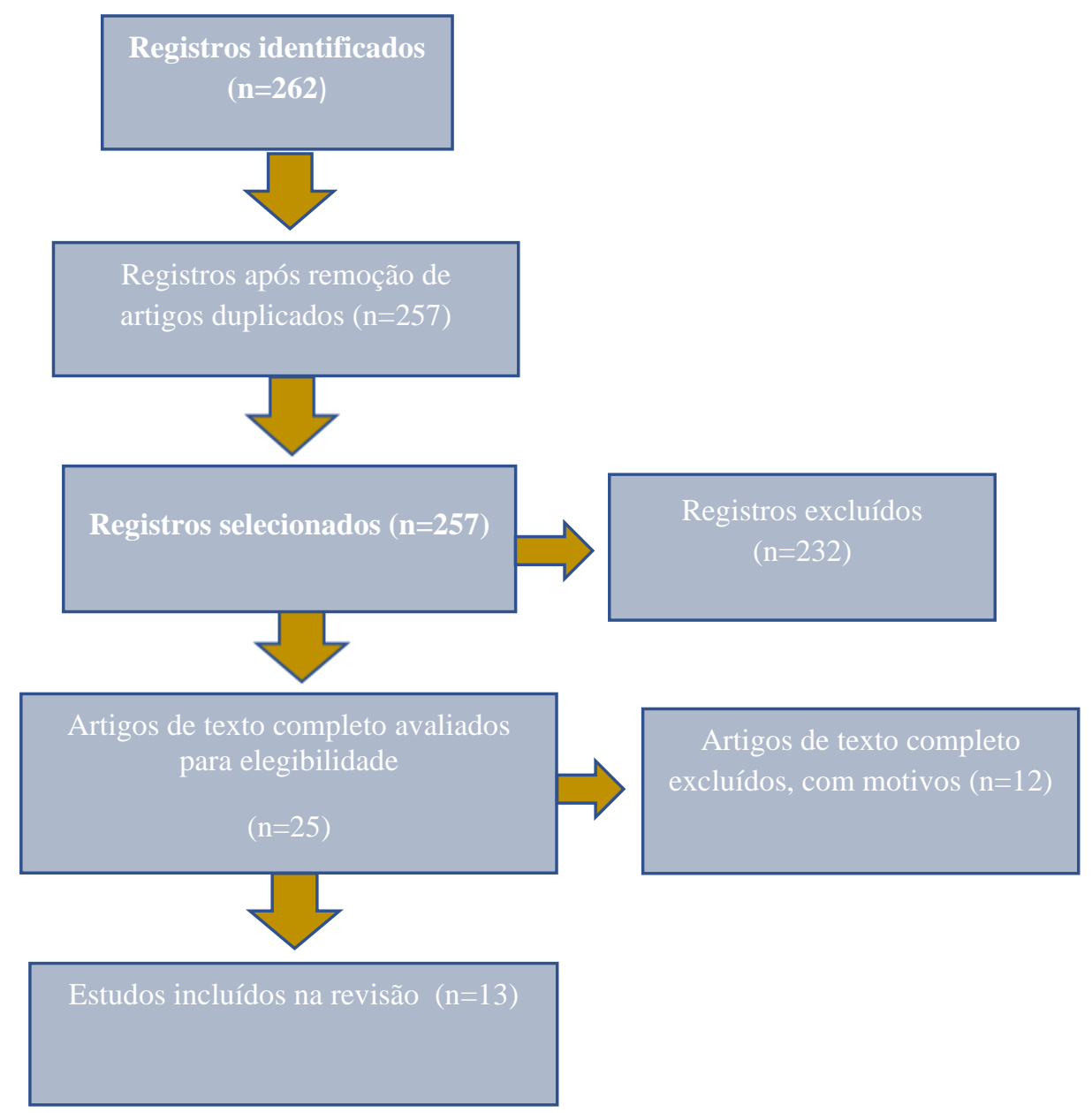

Fonte: Autores (2021). 
Research, Society and Development, v. 11, n.1, e2411124091, 2022

(CC BY 4.0) | ISSN 2525-3409 | DOI: http://dx.doi.org/10.33448/rsd-v11i1.24091

Quadro 1: Resumo dos artigos incluídos na revisão integrativa.

\begin{tabular}{|c|c|c|c|c|c|c|}
\hline Autores & Título & $\begin{array}{c}\text { Local de } \\
\text { busca }\end{array}$ & $\begin{array}{c}\text { Tipo de } \\
\text { pesquisa }\end{array}$ & Descritores & $\begin{array}{c}\text { Ano de } \\
\text { publicação }\end{array}$ & Conclusões/Recomendações \\
\hline $\begin{array}{l}\text { Monique Eva Dias Da Silva; Annie Elisandra } \\
\text { Mesquita de Oliveira; Yolanda de Jesus Morais }\end{array}$ & $\begin{array}{l}\text { Atribuições do farmacêutico no âmbito } \\
\text { hospitalar para promoção da segurança } \\
\text { do paciente: revisão integrativa da } \\
\text { literatura. }\end{array}$ & $\begin{array}{c}\text { Google } \\
\text { acadêmico }\end{array}$ & $\begin{array}{c}\text { Revisão } \\
\text { integrativa }\end{array}$ & $\begin{array}{l}\text { "conciliação } \\
\text { medicamentosa", } \\
\text { "hospital", "Brasil" } \\
\text { e "segurança do } \\
\text { paciente" }\end{array}$ & 2021 & $\begin{array}{l}\text { Todos os estudos demonstram que a presença do } \\
\text { farmacêutico exerce uma interferência benéfica às } \\
\text { prescrições médicas, melhorando assim, a qualidade } \\
\text { dos serviços prestados ao paciente, diminuindo o } \\
\text { óbito, o número e o tempo de hospitalizações e } \\
\text { reduzindo os custos hospitalares. }\end{array}$ \\
\hline $\begin{array}{l}\text { Maria Emília Carneiro de Oliveira; Tiago } \\
\text { Falcão Dias dos Santos; Nadja Layane Gomes } \\
\text { Santiago; Bruno Rodrigues Alencar; Aline } \\
\text { Silva Gomes Xavier; Silvone Santa Bárbara da } \\
\text { Silva }\end{array}$ & $\begin{array}{l}\text { Discrepância de conciliação } \\
\text { medicamentosa na emergência de um } \\
\text { hospital público do estado da Bahia. }\end{array}$ & $\begin{array}{c}\text { Google } \\
\text { acadêmico }\end{array}$ & $\begin{array}{l}\text { Descritivo } \\
\text { com } \\
\text { abordagem } \\
\text { quantitativa }\end{array}$ & $\begin{array}{c}\text { "conciliação } \\
\text { medicamentosa", } \\
\text { "hospital", "Brasil" }\end{array}$ & 2018 & $\begin{array}{l}\text { Concluiu-se que a incidência de discrepâncias de } \\
\text { conciliação medicamentosa no setor de emergências } \\
\text { de um hospital público do estado da Bahia foi total } \\
\text { nos casos analisados e existem falhas na comunicação } \\
\text { entre os trabalhadores da saúde, usuário e familiar. }\end{array}$ \\
\hline $\begin{array}{l}\text { Priscila Abreu Pimenta; Kananda Franciele } \\
\text { Souza Santos; Carla Maria Lima Silva; Iara da } \\
\text { Silva Passos; Izadora Menezes da Cunha } \\
\text { Barros; Giselle de Carvalho Brito. }\end{array}$ & $\begin{array}{l}\text { Conciliação de medicamentos em um } \\
\text { hospital de ensino de Sergipe: lições } \\
\text { aprendidas na implementação de um } \\
\text { serviço. }\end{array}$ & $\begin{array}{c}\text { Google } \\
\text { acadêmico }\end{array}$ & $\begin{array}{c}\text { Ciência da } \\
\text { implementaçã } \\
\text { o }\end{array}$ & $\begin{array}{l}\text { "conciliação } \\
\text { medicamentosa", } \\
\text { "hospital", "Brasil" } \\
\text { e "segurança do } \\
\text { paciente" }\end{array}$ & 2020 & $\begin{array}{l}\text { A promoção de atitudes colaborativas } \\
\text { interprofissionais contribui para maior aceitação das } \\
\text { intervenções farmacêuticas, bem como, uma } \\
\text { ampliação no número de farmacêuticos destinados a } \\
\text { serviços clínicos auxiliará no atendimento da elevada } \\
\text { demanda por serviços de conciliação. }\end{array}$ \\
\hline $\begin{array}{l}\text { Thamires Barboza da Silva; Stella Pegoraro } \\
\text { Alves-zarpelon; João Victor Laureano }\end{array}$ & $\begin{array}{l}\text { Conciliação medicamentosa em uma } \\
\text { unidade de internação de hospital } \\
\text { público do Sul do Brasil. }\end{array}$ & $\begin{array}{c}\text { Google } \\
\text { acadêmico }\end{array}$ & $\begin{array}{l}\text { Transversal, } \\
\text { de caráter } \\
\text { descritivo e } \\
\text { prospectivo }\end{array}$ & $\begin{array}{l}\text { "conciliação } \\
\text { medicamentosa", } \\
\text { "hospital", "Brasil" } \\
\text { e "segurança do } \\
\text { paciente" }\end{array}$ & 2021 & $\begin{array}{l}\text { Os pacientes polimedicados foram os que } \\
\text { apresentaram número maior de possíveis interações } \\
\text { medicamentosas. A identificação dessas } \\
\text { discrepâncias demonstrou a importância da realização } \\
\text { da conciliação medicamentosa e da intervenção do } \\
\text { farmacêutico na prevenção de eventos adversos } \\
\text { relacionados a medicamentos. }\end{array}$ \\
\hline
\end{tabular}


Research, Society and Development, v. 11, n.1, e2411124091, 2022

(CC BY 4.0) | ISSN 2525-3409 | DOI: http://dx.doi.org/10.33448/rsd-v11i1.24091

\begin{tabular}{|c|c|c|c|c|c|c|}
\hline $\begin{array}{l}\text { Calize Oliveira dos Santos; Francieli Zanella } \\
\text { Lazaretto; Lucélia Hernandes Lima; Marcelo; } \\
\text { Schenk Azambuja; Luzia Fernandes Millão }\end{array}$ & $\begin{array}{l}\text { Reconciliação de medicamentos: } \\
\text { processo de implantação em um } \\
\text { complexo hospitalar com a utilização } \\
\text { de sistema eletrônico }\end{array}$ & BVS & $\begin{array}{l}\text { Retrospectivo } \\
\text { e quantitativo }\end{array}$ & $\begin{array}{l}\text { "conciliação } \\
\text { medicamentosa", } \\
\text { "hospital", "Brasil" }\end{array}$ & 2019 & $\begin{array}{l}\text { O acompanhamento diário do farmacêutico clínico } \\
\text { aumenta a segurança do paciente quanto ao uso de } \\
\text { medicamentos dentro dos hospitais, entretanto, para } \\
\text { executar a atividade, é necessário realizar algumas } \\
\text { medidas de melhoria para obter o cumprimento da } \\
\text { reconciliação de medicamentos dos pacientes na sua } \\
\text { totalidade. }\end{array}$ \\
\hline $\begin{array}{l}\text { Diana Domingues da Camara Graça; Walter } \\
\text { Viera Mendes Junior; Saint Clair dos Santos } \\
\text { Gomes Júnior }\end{array}$ & \begin{tabular}{lllr} 
Construção & e & avaliação & de \\
instrumentos & de & \multicolumn{1}{c}{ conciliação } & de \\
medicamentos & para pacientes \\
pediátricos & & &
\end{tabular} & BVS & $\begin{array}{c}\text { Descritivo } \\
\text { prospectivo }\end{array}$ & $\begin{array}{l}\text { "conciliação } \\
\text { medicamentosa", } \\
\text { "hospital", "Brasil" } \\
\text { e "segurança do } \\
\text { paciente" }\end{array}$ & 2018 & $\begin{array}{l}\text { Os formulários de conciliação de medicamentos } \\
\text { construídos e avaliados foram validados para } \\
\text { pacientes pediátricos na instituição estudada e sua } \\
\text { alocação em local visível e acessível de prontuários } \\
\text { de instituições semelhantes permitiria a } \\
\text { disponibilização de relevantes informações sobre os } \\
\text { medicamentos em uso aos profissionais envolvidos } \\
\text { no cuidado de crianças hospitalizadas. }\end{array}$ \\
\hline $\begin{array}{l}\text { Maria Carolina Peçanha Fernandes; Luciana } \\
\text { Favoreto Vieira Mattos; Maria Fernanda } \\
\text { Barbosa. }\end{array}$ & $\begin{array}{lcc}\text { Conciliação } & \text { Medicamentosa } & \text { em } \\
\text { Cuidados Paliativos Oncológicos } & \end{array}$ & $\begin{array}{c}\text { Google } \\
\text { acadêmico }\end{array}$ & $\begin{array}{l}\text { Transversal, } \\
\text { analítico e } \\
\text { descritivo. }\end{array}$ & $\begin{array}{l}\text { "conciliação } \\
\text { medicamentosa", } \\
\text { "hospital", "Brasil" } \\
\text { e "segurança do } \\
\text { paciente" }\end{array}$ & 2021 & $\begin{array}{l}\text { As características dos pacientes em Cuidados } \\
\text { Paliativos valorizam a prática da conciliação } \\
\text { realizada por farmacêuticos, já que se trata de } \\
\text { pacientes polissintomáticos, poliqueixosos e } \\
\text { polimedicados. }\end{array}$ \\
\hline $\begin{array}{l}\text { Zenewton André da Silva Gama ; Pedro Jesus } \\
\text { Saturno-Hernández ; Denise Nieuwenhoff } \\
\text { Cardoso Ribeiro; Marise Reis de Freitas ; Paulo } \\
\text { José de Medeiros ; Almária Mariz Batista; } \\
\text { Analúcia Filgueira Gouveia Barreto; Benize } \\
\text { Fernandes Lira; Carlos Alexandre de Souza } \\
\text { Medeiros; Cilane Cristina Costa da Silva } \\
\text { Vasconcelos ; Edna Marta Mendes da Silva; } \\
\text { Eduardo Dantas Baptista de Faria; Jane } \\
\text { Francinete Dantas; José Gomes Neto Júnior ; } \\
\text { Luana Cristina Lins de Medeiros ; Miguel } \\
\text { Angel Sicolo; Patrícia de Cássia Bezerra } \\
\text { Fonseca; Rosângela Maria Morais da Costa; } \\
\text { Francisca Sueli Monte; Veríssimo de Melo Neto }\end{array}$ & $\begin{array}{l}\text { Desenvolvimento e validação de } \\
\text { indicadores de boas práticas de } \\
\text { segurança do paciente: Projeto ISEP- } \\
\text { Brasil }\end{array}$ & LILACS & $\begin{array}{c}\text { Validação de } \\
\text { instrumentos } \\
\text { de medidas }\end{array}$ & $\begin{array}{l}\text { "conciliação } \\
\text { medicamentosa", } \\
\text { "hospital", "Brasil" } \\
\text { e "segurança do } \\
\text { paciente" }\end{array}$ & 2016 & $\begin{array}{l}\text { Os indicadores foram considerados válidos, } \\
\text { confiáveis e úteis para o monitoramento da segurança } \\
\text { do paciente em hospitais brasileiros. }\end{array}$ \\
\hline $\begin{array}{l}\text { Carlos Eduardo Faria Ferreira; Maurício Assis } \\
\text { Rodrigues; André Assis Rodrigues; Fabiano de }\end{array}$ & $\begin{array}{lr}\text { Identificação de } & \text { reações adversas a } \\
\text { medicamentos } & \text { (RAM) durante }\end{array}$ & $\begin{array}{c}\text { Google } \\
\text { acadêmico }\end{array}$ & $\begin{array}{c}\text { Longitudinal } \\
\text { prospectivo }\end{array}$ & $\begin{array}{c}\text { "conciliação } \\
\text { medicamentosa", } \\
\text { "hospital", "Brasil" }\end{array}$ & 2016 & $\begin{array}{l}\mathrm{O} \text { processo de conciliação de medicamentos } \\
\text { contribuiu para a identificação de RAM, permitindo } \\
\text { ao profissional farmacêutico atuação mais efetiva }\end{array}$ \\
\hline
\end{tabular}


Research, Society and Development, v. 11, n.1, e2411124091, 2022

(CC BY 4.0) | ISSN 2525-3409 | DOI: http://dx.doi.org/10.33448/rsd-v11i1.24091

\begin{tabular}{|c|c|c|c|c|c|c|}
\hline $\begin{array}{l}\text { Souza Santos Filho; Oscar Lara Rocha Junior; } \\
\text { Selma Rodrigues de Castilho }\end{array}$ & $\begin{array}{l}\begin{array}{l}\text { conciliação medicamentosa } \\
\text { hospital escola }\end{array} \\
\end{array}$ & & & $\begin{array}{l}\text { e "segurança do } \\
\text { paciente" }\end{array}$ & & $\begin{array}{l}\text { junto à equipe multiprofissional de saúde no que se } \\
\text { refere às reações indesejáveis causadas pelo } \\
\text { medicamentos possibilitando a prevenção de agravos } \\
\text { relacionados à terapia medicamentosa e açôe } \\
\text { voltadas para a segurança dos pacientes. }\end{array}$ \\
\hline $\begin{array}{l}\text { Lívia Falcão Lima; Bruna Cristina Cardoso } \\
\text { Martins; Francisco Roberto Pereira de Oliveira; } \\
\text { Rafaela Michele de Andrade Cavalcante; } \\
\text { Vanessa Pinto Magalhães; , Paulo Yuri Milen } \\
\text { Firmino; Liana Silveira Adriano; Adriano } \\
\text { Monteiro da Silva; Maria Jose Nascimento Flor; } \\
\text { Eugenie Desirée Rabelo Néri }\end{array}$ & $\begin{array}{l}\text { Orientação farmacêutica na alta } \\
\text { hospitalar de pacientes transplantados: } \\
\text { estratégia para a segurança do } \\
\text { paciente. }\end{array}$ & $\begin{array}{c}\text { Google } \\
\text { acadêmico }\end{array}$ & $\begin{array}{l}\text { Transversal, } \\
\text { descritivo e } \\
\text { retrospectivo }\end{array}$ & $\begin{array}{l}\text { "conciliação } \\
\text { medicamentosa", } \\
\text { "hospital", "Brasil" } \\
\text { e "segurança do } \\
\text { paciente" }\end{array}$ & 2016 & $\begin{array}{l}\text { A orientação do farmacêutico clínico junto à equipe } \\
\text { multiprofissional no momento da alta do paciente } \\
\text { transplantado é importante, pois previne resultados } \\
\text { negativos associados à farmacoterapia, garantindo a } \\
\text { conciliação medicamentosa e a segurança do } \\
\text { paciente. }\end{array}$ \\
\hline $\begin{array}{l}\text { Priscila Helena Marietto Figueira; Renata Rosa } \\
\text { Veloso Cataldo; Elaine Silva Miranda; Gabriela } \\
\text { Bittencourt Gonzalez Mosegui }\end{array}$ & $\begin{array}{l}\text { Método estruturado para a prática de } \\
\text { conciliação medicamentosa em } \\
\text { hospital oncológico. }\end{array}$ & $\begin{array}{c}\text { Google } \\
\text { acadêmico }\end{array}$ & Exploratório & $\begin{array}{l}\text { "conciliação } \\
\text { medicamentosa", } \\
\text { "hospital", "Brasil"" } \\
\text { e "segurança do } \\
\text { paciente" }\end{array}$ & 2019 & $\begin{array}{l}\text { Identificadas discrepâncias; essas foram registrada } \\
\text { de acordo com a classificação de Ote-ro. A partir do } \\
\text { método proposto, os medicamentos utilizados } \\
\text { anteriormente à internação podem ser comparados } \\
\text { com a prescrição médica de internação e as } \\
\text { discrepâncias analisadas, aumentando a qualidade do } \\
\text { cuidado na rotina hospitalar. }\end{array}$ \\
\hline $\begin{array}{l}\text { Mônica Lopes Tonello; Stella Pegoraro Alves; } \\
\text { Márcia de Azevedo Frank; , Denise Bueno }\end{array}$ & $\begin{array}{l}\text { Discrepâncias entre os registros de } \\
\text { prontuários acerca da farmacoterapia } \\
\text { de pacientes pediátricos com fibrose } \\
\text { cística }\end{array}$ & BVS & $\begin{array}{c}\text { Transversal } \\
\text { retrospectivo }\end{array}$ & $\begin{array}{l}\text { "conciliação } \\
\text { medicamentosa", } \\
\text { "hospital", "Brasill" } \\
\text { e "segurança do } \\
\text { paciente" }\end{array}$ & 2017 & $\begin{array}{l}\text { A conciliação tem o objetivo de promover o uso } \\
\text { racional e diminuir os erros relacionados } \\
\text { medicamentos, podendo ser realizada de maneira } \\
\text { sistematizada. A avaliação da qualidade do uso de } \\
\text { medicamentos pode ser feita através da utilização de } \\
\text { indicadores, sendo as discrepâncias um indicador } \\
\text { sensível para este objetivo. }\end{array}$ \\
\hline $\begin{array}{l}\text { Flavio Rodrigues Filho, Paulo Yuri Firmino, } \\
\text { Natália Martins Freire }\end{array}$ & $\begin{array}{l}\text { Avaliação de um serviço de } \\
\text { reconciliação medicamentosa em um } \\
\text { hospital privado de Fortaleza-CE: } \\
\text { indicadores de qualidade }\end{array}$ & $\begin{array}{c}\text { Google } \\
\text { acadêmico }\end{array}$ & $\begin{array}{c}\text { Seccional e } \\
\text { retrospectivo }\end{array}$ & $\begin{array}{c}\text { "conciliação } \\
\text { medicamentosa", } \\
\text { "hospital", "Brasil" } \\
\text { e "segurança do } \\
\text { paciente" }\end{array}$ & 2021 & $\begin{array}{l}\text { A reconciliação de medicamentos no ambiente } \\
\text { hospitalar identificou a necessidade de adição de } \\
\text { medicamentos, como a intervenção mais comum } \\
\text { além da necessidade de comunicar as comorbidades } \\
\text { dos pacientes a equipe médica. }\end{array}$ \\
\hline
\end{tabular}

Fonte: Fonte: Autores (2021). 
O farmacêutico clínico trabalha promovendo a saúde, prevenindo eventos adverso e intervindo quando necessário, nas prescrições para obtenção de resultados clínicos positivos. Continuamente a monitorização farmacoterapêutica, é capaz de reduzir erros relacionados a medicamentos, aumentando com isso a efetividade e minimizando os riscos da farmacoterapia nesse sentido. Este profissional traz benefícios através da identificação de potenciais eventos adversos relacionados à terapia medicamentosa e consequentemente, contribui para a segurança do paciente no desfecho positivo ao usuário (Silva, Oliveira \& Moraes, 2021). Todos os artigos selecionados, dentro da segurança do paciente, das atividades realizadas pelo farmacêutico, demonstram que a presença deste exerce uma interferência benéfica às prescrições médicas, melhorando assim, a qualidade dos serviços prestados ao paciente, diminuindo o óbito, o número e o tempo de hospitalizações e de custos hospitalares.

Silva, Oliveira e Moraes (2021) ressaltam que dentre as atividades realizadas pelos farmacêuticos clínicos estão o processo de avaliação de prescrição de medicamentos antes da dispensação e administração, conforme critérios rígidos, além da prática da CM, validação de suspeitas de reação adversa, promoção da educação aos pacientes e familiares/acompanhantes quanto ao uso de medicamentos. Oliveira et al. (2018) acrescenta que a CM visa garantir medicamentos corretos nas transições dos cuidados, mediante a avaliação da necessidade da continuação ou suspensão dos medicamentos, além de prestar orientações para os cidadãos não permanecerem com os medicamentos usados no domicílio na unidade hospitalar, a fim de evitar duplicação da dose ou o risco do uso de medicamentos não indicados para a condição clínica deste.

O estudo de Oliveira et al.(2018) realizado no setor de emergência de um hospital público no estado da Bahia, verificou a incidência de discrepâncias de CM. Como resultado, observaram que a prática da CM ocorreu em 23,4\% (n=18) dos prontuários para alguns medicamentos e em apenas $6,5 \%(n=5)$ para todos os medicamentos; em $70,1 \%$ dos casos $(n=54)$, a prática não foi realizada. No total foram encontradas 141 discrepâncias, sendo 50,34\% $(n=71)$ que não requeriam esclarecimentos com o prescritor e 49,66\% (n=70) que requeriam esclarecimentos. Os autores concluem que a falta de informação e a comunicação inadequada entre cidadãos, profissionais e familiares na admissão no serviço de emergência apresentam resultados preocupantes, uma vez que, nos prontuários analisados, não continham informações essenciais para a prática da CM, a exemplo de informações sobre medicamentos de uso contínuo, suas respectivas dosagens e horários, alergias, entre outras informações.

O estudo de Da silva, Alvez-Zarpelon \& Laureno (2021) realizado com pacientes internados em unidade clínica de um hospital, corrobora com a importância da CM. Os autores concluíram que a ferramenta de CM possui grandes desafios como por exemplo, a própria intervenção farmacêutica, na realização do contato com a equipe médica para justificativas das alterações do tratamento, o que sinaliza a importância do cuidado multiprofissional ao paciente.

Outro estudo que também incentiva a adoção de CM é o de Fernandes, Matos \& Barbosa (2021), com o objetivo de analisar o perfil das CM em pacientes que estão sob cuidados paliativos oncológicos, foram analisadas todas as visitas de conciliações realizadas na admissão de pacientes. Os autores concluíram que as características dos pacientes em cuidados paliativos valorizam a prática da conciliação realizada por farmacêuticos, pois se trata de pacientes polissintomáticos, poliqueixosos e polimedicados. Logo, o perfil da conciliação não se distância da literatura e, com as informações coletadas no momento da entrevista, o farmacêutico pode atuar no auxílio do manejo dos sintomas ao realizar outras intervenções que não estão inseridas no processo de conciliação.

Pimenta et al. (2020) realizaram um estudo de implementação do serviço de CM, onde 82 pacientes foram atendidos pelo serviço. Foram identificadas discrepâncias na conciliação de 41 (50,00\%) pacientes atendidos, sendo posteriormente realizada intervenção farmacêutica para cada um destes. Neste trabalho os autores enfatizam que a clínica é exclusivamente realizada pelos farmacêuticos residentes, assim, cada semestre apenas umas das residentes realizavam as conciliações na clínica, sem disponibilidade de dedicação exclusiva a essa atividade, limitando o tempo disponibilizado para essa e o número de 
atendimentos. Ademais, a comunicação escrita não foi eficaz e a carência de ações colaborativas foram fatores que limitaram a aceitação das intervenções farmacêuticas.

Corroborando com o estudo acima, Santos et al. (2019) realizaram estudo que avaliou a implantação da CM em um hospital multibloco, filantrópico e de ensino com a utilização de um sistema eletrônico para realizar o registro. Observaram que a utilização de sistemas informatizados pode ser útil para as equipes executarem a CM, dependendo da correta utilização do sistema e treinamento das equipes.

Na mesma vertente, Graça et al. (2018) objetivaram construir e avaliar instrumentos de CM para os momentos da admissão hospitalar e transferência interna de pacientes pediátricos. Enfatizaram que os instrumentos de CM construídos foram avaliados em um único hospital, especializado, com pacientes pediátricos, por isso, seu resultado pode não ser generalizável. Sendo interessante a construção e avaliação dos instrumentos de CM em outros serviços, contextos e grupos de pacientes.

Em um estudo de Gama et al. (2016), relataram que um monitoramento eficaz da segurança do paciente precisa focar a implantação de práticas baseadas em evidências que evitem danos desnecessários, ligados à assistência à saúde. Logo, esclareceram que os indicadores desenvolvidos e validados neste estudo, constituem uma contribuição para a gestão da segurança do paciente no contexto dos hospitais brasileiros. O modelo de monitoramento de eventos adversos pode ser considerado como o enfoque epidemiológico da segurança do paciente. Enfatizam que futuras pesquisas podem verificar o nível de implementação das práticas seguras, estratégias para melhorar a adesão, influência do contexto na implementação (liderança, cultura organizacional, tamanho dos hospitais, tipo de gestão pública ou privada, financiamento, entre outros).

Ferreira et al. (2016), acrescentam que a CM contribui de forma significativa para que o profissional farmacêutico se mantenha informado e vigilante a respeito das reações indesejáveis, causadas pelos medicamentos, possibilitando a prevenção de agravos relacionados à terapia medicamentosa. Além disso, permite uma maior integração deste profissional com a equipe multidisciplinar composta por médicos, enfermeiros, nutricionistas entre outros, aumentando as ações voltadas para a segurança dos pacientes. Lima et al. (2016), complementam que a alta hospitalar de pacientes transplantados é um processo que envolve toda a equipe multiprofissional. O farmacêutico é responsável pela orientação do tratamento medicamentoso e deve elaborar o plano de alta, levando em conta as particularidades do paciente, a fim de que o planejamento seja adequado para a realidade de cada indivíduo.

Figueira et al. (2019), realizando o método de internação de pacientes, objetivaram a criação de um método para a prática de CM de forma estruturada. A partir do método proposto, os medicamentos utilizados anteriormente à internação podem ser comparados com a prescrição médica de internação e as discrepâncias analisadas, aumentando a qualidade do cuidado na rotina hospitalar. A qualidade dos resultados de um processo de CM está diretamente relacionada à qualidade dos dados coletados. Assim, um instrumento informativo e bem estruturado simplifica o design do banco de dados e a validação destes.

Tonello et al. (2017) estudaram a discrepâncias entre os registros de prontuários acerca da farmacoterapia de pacientes pediátricos com fibrose cística. Foram observadas omissões de dose no registro farmacêutico e no médico e omissões de regime em ambos. Concluíram que a CM tem o objetivo de promover o uso racional e diminuir os erros relacionados a medicamentos, podendo ser realizada de maneira sistematizada. A avaliação da qualidade do uso de medicamentos pode ser feita a partir da utilização de indicadores, sendo as discrepâncias um indicador sensível para este objetivo.

Filho et al. (2021), em estudo realizado em um hospital privado, observaram que a quantidade total de medicamentos conciliados e aceitos foi de 228, havendo conciliação de 36 classes terapêuticas e o nível de aceitação das intervenções foi considerado alto $(77,5 \%)$. A intervenção de maior número foi a de adição de medicamentos, ressaltando assim as falhas em relação a comunicação sobre os medicamentos que o paciente estava fazendo uso anterior à sua internação. 


\section{Considerações Finais}

A segurança do paciente se define como o ato de evitar, prevenir ou melhorar os resultados adversos ou lesões originadas no atendimento médico-hospitalar, se tornando então uma questão de saúde pública, tendo em vista que os riscos e eventos que podem ocasionar qualquer prejuízo ao paciente estão presentes em diversos ambientes, principalmente o ambiente hospitalar (Filho et al. 2021).

Com base no levantamento de dados, observou-se que a conciliação tem o objetivo de promover o uso racional e diminuir os erros relacionados a medicamentos, podendo ser realizada de maneira sistematizada. Sabe-se que a falta de precisão nos registros de farmacoterapia ambulatorial, quando persiste, pode contribuir para propagar as discrepâncias em outros níveis de atenção à saúde, como a admissão hospitalar, pois muitas dessas informações são transmitidas de um serviço para outro. Sem uma verificação adequada dos fármacos em uso pelo paciente, há maiores chances de falhar no diagnóstico e detecção de problemas relacionados com medicamentos.

Nesse contexto, o papel do farmacêutico é fundamental para uma melhora na qualidade de vida dos pacientes internados em hospitais, pois ele pode esclarecer dúvidas quanto aos medicamentos, quanto a sua doença, podendo viabilizar meios para a adesão ao tratamento medicamentoso, orientar quanto à necessidade, os riscos e os benefícios da medicação e promover o uso racional destes. Os resultados obtidos relataram a significância da CM dentro do ambiente hospitalar, apresentando-se como uma ótima ferramenta com a participação do farmacêutico no cuidado clínico ao paciente.

Em todos os artigos analisados, a CM apresentou-se como fundamental para a segurança do paciente, com elevada importância de se monitorar as prescrições e trabalhar em conjunto com a equipe multiprofissional. Portanto, a conciliação medicamentosa é uma ferramenta positiva para a saúde da população. Contudo, observou-se carência em estudos sobre essa temática, havendo a necessidade de ampliação desse serviço farmacêutico e de desenvolvimento de estudos científicos que abordem a CM com intuito de contribuir para literatura e principalmente segurança do paciente no ambiente hospitalar.

\section{Referências}

Batista, A. V. A.; Dos santos, V. R. C. ; Carneiro, I. C. R. S. (2021). Cuidado farmacêutico em oncologia: Revisão integrativa da literatura. Research, Society and Development,14(1), 1-14.

Silva, M. E. D.; De Oliveira, A. E. M; Morais, Y. J. (2021). Atribuições do farmacêutico no âmbito hospitalar para promoção da segurança do paciente: revisão integrativa da literatura. Research, Society and Development, 10( 13),1-13.

Oliveira, M. E. C.; Dos Santos, T. F. D.; Santiago, N. L. G.; Alencar, B. R.; Xavier, A. G.; Da Silva, S. S.(2018). Discrepância de conciliação medicamentosa na emergência de um hospital público do estado da Bahia. Revista Baiana de Saúde Pública, 42 (1), 127-144

Fernandes, M. C. P.; Mattos, L. F. V.; Barbosa, M. F.(2021). Conciliação Medicamentosa em Cuidados Paliativos Oncológicos. Revista Brasileira de Cancerologia 67(4): 1-7.

Ferreira, C. E. F.; Rodrigues, M. A.; Rodrigues, A. A.; Filho, F. S. S.; Junior, O. L. R.; De Castilho, S. R. (2016). Identificação de reações adversas a medicamentos (RAM) durante conciliação medicamentosa em hospital escola. Cienc Cuid Saude ; 15(3):445-451.

Filho, F. R.; Firmino, P. Y. Natália Martins Freire, N. M. (2021). Avaliação de um serviço de reconciliação medicamentosa em um hospital privado de FortalezaCE: indicadores de qualidade. Bras Farm Hosp Serv Saude., 12(1): 1-7

Figueira, P. H. M.; Cataldo, R. R. V.; Miranda, E.S.; Mosegui, G.B.G. (2019). Método estruturado para a prática de conciliação medicamentosa em hospital oncológico. Sistemas \& Gestão 14 (3), 1-8.

Gama, Z. A. S.; Saturno-Hernández, P. J.; Ribeiro, D. N. C.; De Freitas, M .R ; De Medeiros, P. J. ; Batista, A. M.; Barreto, A L. F. G.; Lira, B. F.; Medeiros, C. A. S.; Vasconcelos, C. C. C. S. ; Da Silva, E. M. M.; De Faria, E. D. B.; Dantas, J. F.; Júnior, J. G. N. ;De Medeiros, L. C. L. ; Sicolo, M. A.; Fonseca, P. C. B.; Da Costa, R. M. M.; Monte, F. S.; Neto, V. M. (2016). Desenvolvimento e validação de indicadores de boas práticas de segurança do paciente: Projeto ISEPBra. Cad. Saúde Pública, 32(9): 1-17.

Graça , D. D. C; Junior, W. V. M; Junior, S. C. S. (2018). Construção e Avaliação de Instrumentos de Conciliação de Medicamentos para pacientes pediátricos Rev. Bras. Farm. Hosp. Serv. Saúde, 9(4): 1-8. 
Research, Society and Development, v. 11, n.1, e2411124091, 2022

(CC BY 4.0) | ISSN 2525-3409 | DOI: http://dx.doi.org/10.33448/rsd-v11i1.24091

Lima, L. F.; Martins, B. C. C.; De Oliveira, F. R. P.; Cavalcante, R. M. A.; Magalhães, V. P.; Firmino, P. Y. M.; Adriano, L. S, Da Silva, A. M.; Flor; M. J. N. ; Neri, E. D. R. (2016). Orientação farmacêutica na alta hospitalar de pacientes transplantados: estratégia para a segurança do paciente. Einstein. ,14(3):359-65

Magalhães, G.F. (2017). Conciliação medicamentosa em pacientes de um hospital Universitário (Dissertação de mestrado, Universidade Federal da Bahia)

Pimenta, P. A.; Santos, K. F. S.; Silva, C. M. L.; Passos, I. S.; Barros, I. M. C.; Brito, G. C. (2020). Conciliação de medicamentos em um hospital de ensino de Sergipe: lições aprendidas na implementação de um serviço. Scientia Plena. 16(8), 1-13.

Santos, C. O.; Lazaretto, F. Z.; Lima, L. H.; Azambuja, M. S.; Fernandes, L. (2019). Reconciliação de medicamentosa: processo de implantação em um complexo hospitalar com a utilização de um sistema eletrônico. Saúde debate, 43(121), 368-377

Silva, T. B.; Alvez-zarpelon, S. P.; Laureano, J. V. (2021). Conciliação medicamentosa em uma unidade de internação de hospital público do Sul do Brasil. Infarma, $33,158-166$

Silvestre, C. C; Júnior, D. L. (2018). Os três C's da conciliação de medicamentos: realidade e perspectivas. Rev. Bras. Farm. Hosp. Serv. Saúde, 9(1): 1-2

Souza, L B S; Souza, D. M; Souza, S. M.; Silva, D. R.; Aguilar, N. C, Importância Do Farmacêutico Clínico No Uso Seguro E Racional De Medicamentos No Âmbito Hospitalar, Pensar Acadêmico, 16(1), 109-124

Tavares, M. K. (2019). Análise de conformidade do processo de conciliação medicamentosa em uma unidade de internação cirúrgica de um hospital universitário. (Residência Multidisciplinar. Universidade Federal de Santa Catarina).

Tonello, M. L.; Alves, S. P.; Frank, M. A.; Bueno, D. (2017). Discrepâncias entre os registros de prontuários acerca da farmacoterapia de pacientes pediátricos com fibrose cística. Clin Biomed Res;37(3), 181-186. 